\title{
PHASE DIAGRAM OF THE STRONG INTERACTION SYSTEM FROM LATTICE QCD
}

\author{
SEYONG KIM \\ Department of Physics, Sejong University, Seoul 143-747, South Korea \\ skim@sejong.ac.kr
}

We briefly review recent progresses in studying QCD thermodynamics from lattice QCD. Investigation of QCD in zero baryon density shows a rapid cross-over with realistic $(u, d, s)$ quark masses. Various improvements of lattice QCD action leads to more accurate determination of QCD thermodynamic properties. Although simulating QCD in nonzero baryon density is difficult due to "sign problem", steady progress is also achieved.

Keywords: QCD; phase diagram; lattice gauge theory.

PACS Nos.: 12.38.-t,12.38.Gc,11.11.Ha

\section{Introduction}

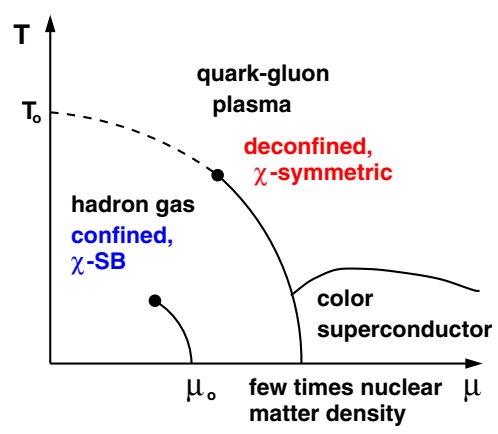

Fig. 1. A schematic phase diagram of the strong interaction system.

A system influenced by the strong interaction is expected to show rich "phase" structure depending on the temperature and the quark number density. Ordinarily(i.e., in zero temperature and in zero net nucleon density), one observes only color singlet states such as meson and baryons instead of fundamental constituents of nature, quarks and gluons. It is because the strong force is "confining". Another interesting property of the strong interaction is "spontaneous chiral symmetry breaking". Chiral partner states have different masses and there exist ligh mesons such as 
$\pi, K$, and etc., even though the strong interaction itself is chirall-symmetric. This phase is called "hadronic" phase. As the system temperature is increased while the quark density is kept to zero, the strong interaction loses its confing nature and

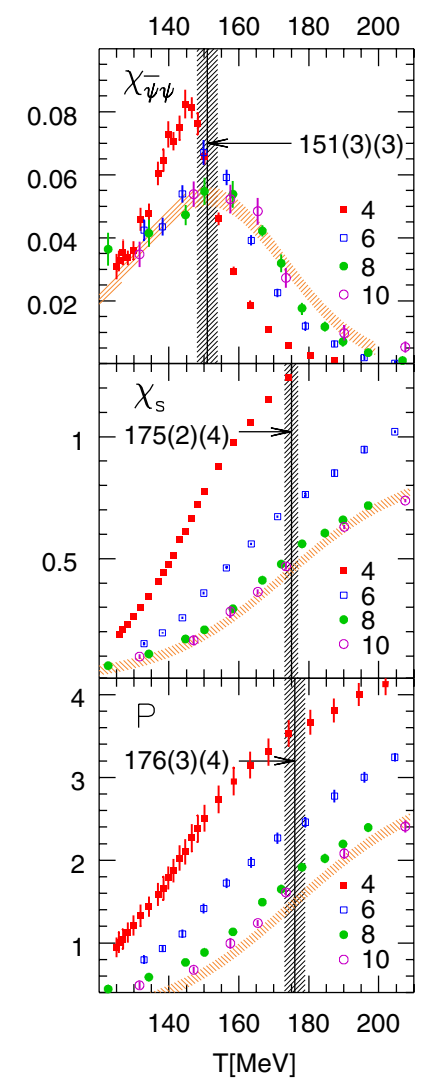

Fig. 2. Behavior of various susceptibilities vs. temperature.

quark-gluon can exist as isolated states. Naturally, the chiral symmetry of the strong interaction manifests itself in the states and the spontaneously broken chiral symmetry gets restored. Another interesting property, "asymptotic freedom", of the strong interaction is that the strength of the interaction grows weak as typical energy scale of the system is increased. Like high electron density in a metal produces "Fermi energy scale" due to the fact that electrons obey Fermi-Dirac statistics, increasing quark number density creates high energy scale. Effective strength of the interactions among small excitation around this energy scale is weakly attractive and color superconducting state similar to metalic superconductor may appear. These phases 
may exist in early universe or may be re-created in laboratory experiments with heavy ions.

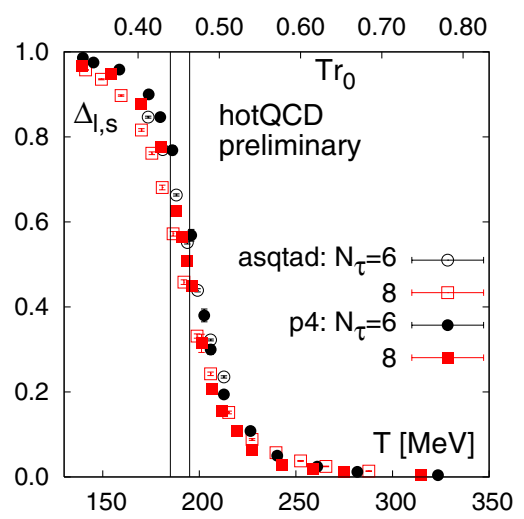

Fig. 3. Behavior of strange quark chiral condensate vs. temperature.

If there are many different macroscopic phases, many questions arise: what are the physical properties of these phases? what is the order of phase transition? where is the phase transition located? Answering these questions concerned with the strong interaction requires non-perturbative techniques because the strong interaction is literally "strong" and model studies are diffcult without sufficient experimental data. Lattice Quantum ChromoDynamics(QCD) offers systematic and quantitative study based on the first principles of quantum field theory and much progress has been made in understanding thermodynamic properties of the strong interaction. ${ }^{1}$

Here, we review recent progresses in this area of research. Let us remind that a result from lattice study has three sources of systematic errors: finite quark mass, finite lattice spacing, and finite lattice volume. Present studies employ almost physical quark masses: $u, d$ quark masses used in the simulation is about twice of corresponding current quark masses and $s$ quark masses is similar to its current quark mass. In principle, lattice spacing is controlled by the gauge coupling. Any lattice action suffices for a numerical study as long as the proper continuum limit is achieved. Recent studies use various "improved" lattice actions each of which improve different aspects of finite lattice spacing effect. Of course, each improved lattice actions have different scaling behavior. To express lattice result in terms of physical MeV scale, simulation which can be used for scale determination has to be performed separately. Also, thermodynamic limit means that the spatial extent of space-time lattice should be larger than the time extent, which limits the number of lattice sites along the time direction $\left(N_{t}\right)$ used in lattice simulations because overall lattice space-time should be small enough to fit the capability of modern computers. Currently $N_{t}$ is limited to $\leq 12$ and how fast the continuum limit can be reached is an important problem. 
Figure 1 is a schematic QCD phase diagram. ${ }^{2}$ The quark number density can be controlled by the quark chemical potential $(=\mu)$ and the horizontal axis in Fig. 1 is in unit of the quark chemical potential.

\section{Finte Temperature, $\mu=0$}

Lattice QCD study in finite temperature and zero quark number density show us that the strong interaction system with physical $(u, d)$ mass and physical $s$ mass has a rapid cross-over finite temperature transition. Where is this cross-over transition located? Since a cross-over is not really a phase transition but a rapid change of order parameters in the narrow range of thermodynamic variables, each physical quantities may show different behaviors.

Indeed, Fig. $2^{3}$ shows different transition temperature. $(u, d)$ quark chiral susceptibilities shows 151(3)(3) MeV transition temperature and $s$ quark chiral susceptibilities and Polyakov line show 175(2)(4) MeV and 176(3)(4) MeV transition temperature respectively. This study uses so called $p 4$ fat3-improved lattice action. On the other hand, recent study by HoTQCD collaboration which use both $p 4$ fat3-improved action and asqtad-improved lattice action ${ }^{4}$ gives different transition temperature. Preliminary result of ${ }^{4}$ shows $190(5) \mathrm{MeV}$. To fix the lattice spacing scale, these two studies use different physical quantities to make direct comparision between the two results difficult.

Unlike sharp transition like a first phase transition or a critical phase transition, locating a rapid cross-over transition temperature is difficult and there are many sources of uncertainty. ${ }^{3}$ In particular, various improved lattice actions introduces different systematics in scale determination. Further careful study is needed.
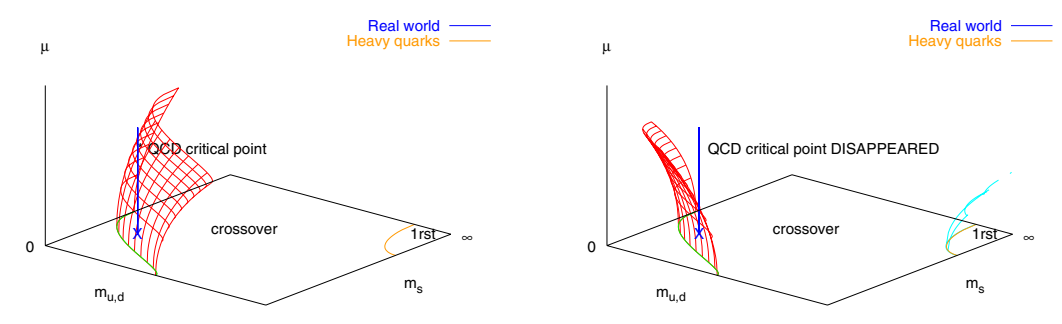

Fig. 4. The possible shape of the critical surface in the $\left(m_{u, d}, m_{s}, \mu\right)$ space. For one possibility, there exists the critical end-point(left Fig.). For another, the critical end-point does not exist(right Fig.). 


\section{Finite Temperature, $\mu \neq 0$}

Studying along the horizontal axis, i.e., environment with fixed temperature and changing quark number density using lattice QCD is difficult unlike the situation in the previous section, environment with changing temperature and zero quark number density, because lattice QCD action with finite quark number density becomes complex( hermitian bases which makes both fermion number density term and gauge interaction term real can not be found) and numerical simulation with complex action has an oscillatory behavior. Fortunately, the parameters space of high temperature-small quark chemical density can be investigated by various methods. ${ }^{5}$

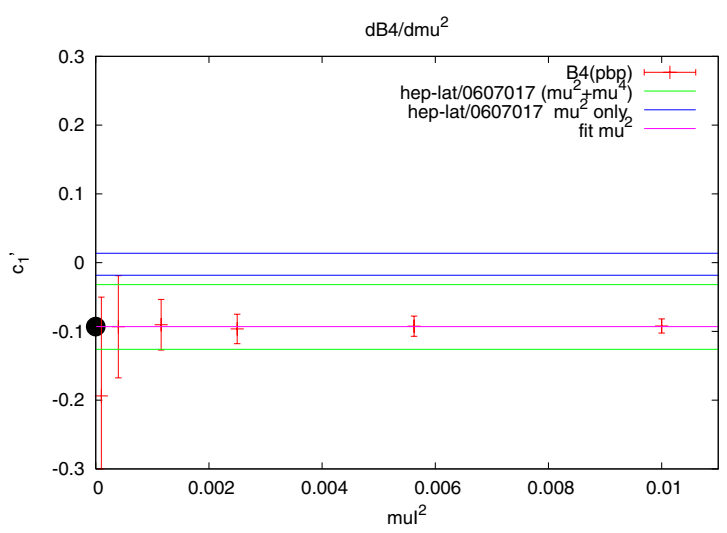

Fig. 5. The slope along the $\mu$-axis.

An interesting question is whether the rapid cross-over transition discussed in the previous section changes into different kind of phase transition. If cross-over changes into a first order transition, QCD phase transition will accompany latent heat. If cross-over changes into a critical transition, there will be critical fluctuation due to diverging correlation length. Either possibility implies interesting phenomena in the evolution of early universe.

Various theoretical considerations suggest a first order phase transition at zero temperature but at finite quark chemical potential. Thus, it is plausible that there exists a critical end-point in Fig. 1. In $\left(\mu, m_{u, d}, m_{s}\right)$ space, this implies the left picture in Fig. 4. On the other hand, recent study by ${ }^{6}$ suggests the right picture in Fig. 4. Critical line with $\mu=0$ can be identified reliably. On this line, the slope at $\mu=0$ along the $\mu$ axis can be determined without the complex action problem because the quark number density is zero at this point. Fig. 5 is the slope from Ref. 6. It is small but definitely negative, which implies that the critical surface from $\mu=0$ plane in $\left(\mu, m_{u, d}, m_{s}\right)$ space curves away from the vertical surface. A study ${ }^{7}$ based on 3-dimensional $Z(3)$-Potts model strengths this observation. In Ref. 7, the slope of 3-D Potts model which is equivalent to QCD with heavy quark mass is 
positive, which is depicted in the right hand corner of Fig. 4. However, the study in Ref. 6 uses coarse lattices $\left(N_{t}=4,6\right)$ compared to the finite temperature QCD studies $\left(N_{t} \leq 12\right)$ and similar study on a finer lattice is needed.

\section{Conclusion}

Thermodynamically, QCD has many interesting macroscopic phases, hadronic state, quark-gluon plasma state, and color superconducting state. Lattice QCD allows us to study quantititively many non-perturbative features of these phases and phase transitions between them. In particular, finite temperature transition can be studied in detail. System with $u, d, s$ quark has a rapid cross-over transition. More accurate determination of transition temperature in continuum limit needs understanding on systematic errors and further studies are under way.

Phase transition associated with quark number density is more difficult due to the complex action problem. The current status of lattice QCD simulation is more qualitative than quantitative. Establishing the existence of critical end-point in $(T, \mu)$ space is important due to its phenomenological implication. Recent study on the slope of the critical surface in $\left(\mu, m_{u, d}, m_{s}\right)$ space at the $\mu=0$ plane shows a small negative slope, which suggests that the critical end-point may not exist. Further investigation in continuum limit and larger space-time lattice is necessary.

\section{Acknowledgments}

This work is supported by the Korea Research Foundation under MOEHRD Basic Research Promotion Grant No. KRF-2006-311-C00020.

\section{References}

1. See for example, proceedings of annual "International Symposium on Lattice Field Theory", on http://pos.sissa.it.

2. M. Stephanov, PosLAT2006:024, 2006, hep-lat/0701002.

3. Z. Fodor, hep-lat/07110336.

4. R. Gupta, arXiv-0810.1764.

5. Z. Fodor and S. D. Katz, Phys. Lett. B 534 (2002) 87 [arXiv:hep-lat/0104001]; Z. Fodor and S. D. Katz, JHEP 0203 (2002) 014.

C. R. Allton et al., Phys. Rev. D 66 (2002) 074507; C. R. Allton, S. Ejiri, S. J. Hands, O. Kaczmarek, F. Karsch, E. Laermann and C. Schmidt, Phys. Rev. D 68 (2003) 014507.

P. de Forcrand and O. Philipsen, Nucl. Phys. B 642 (2002) 290; M. D'Elia and M. P. Lombardo, Phys. Rev. D 67 (2003) 014505.

6. Ph. de Forcrand, PosLAT2007:178,2007, arXiv-0711.0262.

7. S. Kim and Ph. de Forcrand, PosLAT2005:166, 2006, hep-lat/0510069. 